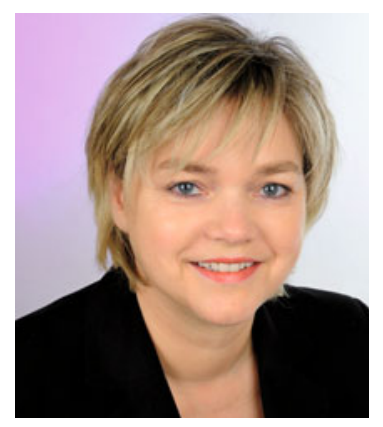

Andrea Tauchert, Berlin

Leitung Kongressbüro andrea.tauchert@springer.com

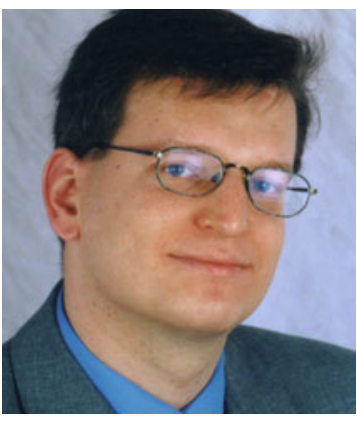

Prof. Dr. med. habil. Jörg Klewer, Zwickau

Schriftleitung

heilberufescience@springer.com

HeilberufeSCIENCE 2011; 2 (Suppl. 1): 2 DOI 10.1007/s16024-011-0101-0

\title{
Kongress „Pflege 2011“
}

Mit dieser ersten Sonderausgabe 2011 zum Kongress „Pflege 2011“ am 28. und 29. Januar 2011 in Berlin bieten wir Ihnen in HeilberufeSCIENCE den freien Zugang zu den Abstracts der Fachvorträge und Workshops. Aufgrund der umfangreichen thematischen Spannbreite und den erfahrungsgemäß mehr als 1.000 Fachbesuchern gehört dieser Kongress zu den größten Fortbildungsveranstaltungen in der Pflegebranche.

Infolge der großen Nachfrage nach den Abstracts zum Kongress "Pflege 2010", haben wir uns entschlossen, diese erfolgreiche Reihe fortzusetzen. Damit hat sich die inhaltliche Verbindung zwischen den regulären vierteljährlichen HeilberufeSCIENCE-Ausgaben und den HeilberufeKongressen weiter positiv entwickelt. Somit werden sowohl der Theorie-PraxisTransfer gestärkt, als auch in der gemeinsamen Diskussion auf den Kongressen für praktische Probleme mit wissenschaftlichen Methoden entsprechende Lösungen gefunden.

Wie schon im Jahr 2010 ersetzt auch diese HeilberufeSCIENCE-Sonderausgabe die bisher übliche Kongress-CD mit den Abstracts und erleichtert somit vielen Interessierten aus Forschung und Praxis die Recherche sowie den Zugang. Indem nun die Inhalte in einer zitierfähigen Version vorliegen, steigert dies die Bedeutung der einzelnen Kongressbeiträge und bietet Anknüpfungspunkte für weitere wissenschaftliche Projekte.

Diese HeilberufeSCIENCE-Sonderausgabe umfasst zwar nicht alle, aber die Abstracts der meisten Vorträge und Workshops in
Berlin. Alphabetisch gegliedert erstrecken sie sich über die Themenfelder Pflegerecht, Pflegemanagement und Pflegepraxis. Es finden sich Beiträge über Servicekräfte in der Pflege genauso wie solche zur Neuordnung von Aufgaben, zur multiprofessionellen Zusammenarbeit oder Patientensicherheit.

Wie immer möchten wir Sie auffordern, HeilberufeSCIENCE kritisch zu lesen, um Gehörtes noch einmal zu vertiefen oder Neues zu entdecken. Wir freuen uns über Ihr Interesse, Ihre Anregungen - Ihr Feedback ist willkommen.

Ihre

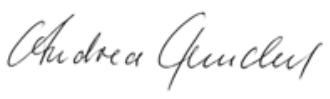

Ihr

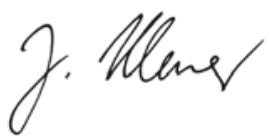

